\title{
PHOTON-PHOTON INTERACTIONS
}

\author{
Frederick J. Gilman
}

\author{
Stanford Linear Accelerator \\ Stanford University, Stanford, California 94305
}

Abstract: A brief summary of the present status of photon-photon interactions is presented. Stress is placed on the use of two-photon collisions to test present ideas on the quark constituents of hadrons and on the theory of strong interactions.

\section{Introduction}

Photons interact by coupling to electric charge. Although then there is no direct photon-photon interaction in the Lagrangian for electrodynamics, we have the process

$$
r \gamma \rightarrow e^{+} e^{-}
$$

in Quantum Electrodynamics, which provides an absorptive part to the photon-photon scattering amplitude. The corresponding dispersive part of the amplitude is due to the "box diagram" with an electron running around the closed loop. In a somewhat different language this was already known and understood in the 1930's soon after Quantum Field Theory was invented. The first calculations ${ }^{1,2}$ ) date from this same period of roughly 45 years ago.

So why is there such a revival of interest in this old subject of photonphoton interactions now? The answer is partly theoretical, but mostly experimental. There are many ways to envision photon-photon collisions: scattering a photon beam on a photon in the Coulomb field of a nucleus (Primakoff effect), shining a laser at a high energy photon beam, etc. But most importantly photon-photon collisions are an automatic by-product of building high-energy electron-positron colliding beam machines. There they are seen as being due to both the initial electron and positron emitting almost real photons which collide to produce a set of particles of invariant mass $M$. If the energy of the colliding (electronpositron) beams, $\mathrm{E}$, is much greater than $\mathrm{m}_{e}$ and we work in the "equivalent photon approximation," then the cross section (integrating over the final electron and positron) ${ }^{3}$ ) is

where

$$
\sigma(e e+e e X)=2\left(\frac{\alpha}{\pi} \ln \frac{E}{m_{e}}\right)^{2} \int^{4 E^{2}} \frac{d^{2}}{M^{2}} f\left(\frac{M}{2 E}\right) \sigma(\gamma \gamma \rightarrow X),
$$

$$
f(x)=\left(2+x^{2}\right)^{2} \ln \left(\frac{1}{x}\right)-\left(1-x^{2}\right)\left(3+x^{2}\right) \text {. }
$$

The most notable aspect of Eq. (1) for the present discussion is the energy dependence. Unlike the one photon annihilation cross section which falls as $1 / \mathrm{E}^{2}$, $\sigma(e e \rightarrow e e X)$ rises logarithmically. The two photon process always "wins" at sufficiently high energy, even though it is intrinsically of higher order in the fine structure constant. This was one of the important points brought out a decade ago when the subject was reborn ${ }^{4}$ ) just at the time $e^{+} e^{-}$annihilation through onephoton into multihadrons was first being investigated. At a beam energy of $15 \mathrm{GeV}$, the cross section for one-photon annihilation is a few tenths of nanobarns. That for two-photon production of hadrons is greater than ten nanobarns. While this striking lifference still does not prevent one from clearly separating one-photon annihilation events experimentally by various cuts, it does mean that two-photon processes are comparatively common at the present generation of $\mathrm{e}^{+} \mathrm{e}^{-}$storage rings and capable of serious study.

* Work supported by the Department of Energy, contract DE-AC03-76SF00515. (Invited talk presented at the 9 th International Conference on the Few Body Problem, Eugene, Oregon, August 17-23, 1980.) 


\section{Testing Quantum Electrodynamics}

The simplest, and original ${ }^{1}$ ), two-photon process is the production of a lepton pair

$$
\begin{aligned}
& \text { ee } \rightarrow \text { ee } e^{+} e^{-} \\
& \text {ee } \rightarrow \text { ee } \mu^{+} \mu^{-}
\end{aligned}
$$

There have been many observations of these processes and the Quantum Electrodynamics (QED) calculations have been carried out in great detail. ${ }^{3}$ ) Needless to say everything agrees within the (generally 10 to 20\%) experimental errors with QED. The recent experiments at PETRA ${ }^{5}$ ) bring the data up to a produced lepton pair mass $M \approx 4 \mathrm{GeV}$.

\section{Two photon production of hadrons}

The transformation required to go from the lepton production of the last section to production of hadrons is extremely simple: replace the produced lepton pair by a quark-antiquark pair. As long as we neglect both quark and lepton masses, the ratio of cross sections

$$
\frac{\sigma\left(e e+e \bar{q}_{i} q_{i}\right)}{\sigma\left(e e+e \mu^{+} \mu^{-}\right)}=\sum_{\substack{\text { quark } \\ \text { types }}}\left(\frac{e_{i}}{e}\right)^{4},
$$

with. neglect of all the corrections due to strong interactions between the quarks. The sum over quark types on the right-hand side of $\mathrm{Eq}$. (3) not only involves a sum over flavors (up, down, strange, charm, ...) as they are relevant to the particular process under consideration, but also over the three colors (for each. flavor) a quark can carry.

The theory of strong interactions is thought to be one involving vector gatige bosons ("gluons") interacting with quarks by coupling to their color charge. This theory of Quantum Chromodynamics (QCD) is constructed in analogy to QED except it is nonAbelian: the gluons themselves carry color and interact among themselves. In general there are nontrivial corrections due to QCD to a lowest order quark process such as ee $\rightarrow$ eeq. . The goal is to find situations where these corrections are negligible, or if not negligible, where they are computable and/or controllable. As such, the situation here is much like that in other parts of high-cnergy physics where the physics of quarks and QCD are being explored. The advantage we have over, say, studying a purely hadronic collision, is that we start with a known coupling of the photon to the quark. What follows are four examples of how this is pursued in hadron production by two-photons.

\subsection{PRODUCTION OF RESONANCES BY TWO-PHOTONS}

The quark-antiquark pair is bound-up into a meson resonance in this situation. The amplitude is then proportional to the square of the charge of the quark involved, times a factor dependent on the $\bar{q} q$ wave function of the resonance. While proposed ${ }^{6}$ ) for measuring the $\pi^{0}$ width originally, it is only in the last year or so that the first resonance width into $\gamma \gamma$ was measured ${ }^{7}$ ) in this way - that of the $n^{\prime}$. More recent data 5,8 ) show evidence of the $f$ and provide upper limits on the $A_{2}$ and $f^{\prime}$ coupling to $\gamma \gamma$. Evidence ${ }^{9}$ ) for the decay of the charmonium state, $X_{2}(3550) \rightarrow \gamma \gamma$, indicates it should be produced in two-photon collisions as well.

If we know something about the wave function of the resonance (or a relation between wave functions of a family of states such as $\left.\pi^{\circ}, \eta, \eta^{\prime}\right)$, then we gain information from the $\mathrm{Yr}$ coupling about the charge of the quarks inside. Conversely, if we know the quark content, we gain knowledge of the wave function ${ }^{0}$ ). 


\subsection{JETS}

At high enough transverse momenta (to the incident photons) the quark and antiquark in the basic process

$$
\gamma \gamma \rightarrow \bar{q} q
$$

should become manifest ${ }^{1}$ ) as observable jets composed of the hadrons into which they fragment. Analogous jets have been seen in $e^{+} e^{-} \rightarrow \bar{q} q$ experiments a number of years ago. Furthermore the cross section is predictable as just $\Sigma\left(e_{i} / e\right)^{4} \times$ $\sigma\left(\gamma \gamma+\mu^{+} \mu^{-}\right)$. The first evidence for these jets is now being seen from experiments at PETRA $\left.{ }^{12}\right)$.

\subsection{EXCLUSIVE CHANNELS}

The cross sections for processes like $\gamma \gamma \rightarrow \pi \pi, \rho \rho, \ldots$ in the fixed angle, high-energy limit for given charge and helicity states have been predicted ${ }^{3}$ ). Both the energy dependence and absolute rate are calculated in terms of other known quantities.

\subsection{DEEP INELASTIC SCATTERING ON A PHOTON TARGET}

In this case we go to a different kinematic regime: one photon far of the mass-shell with large energy and momentum transfer squared, $Q^{2}$, and the other photon almost real. If we think of this latter, almost on-shell photon as the target, then we have deep inelastic scattering on a photon target. Replacing the set of final hadrons of mass $M$ by a sum of the possible quark-antiquark pairs, one finds a structure function (to which the cross section is proportional) for this process ${ }^{14}$ )

$$
F\left(x, Q^{2}\right)=\frac{\alpha}{2 \pi}\left[x^{2}+(1-x)^{2}\right] \text { ln } Q^{2} \sum_{i}\left(e_{i} / e\right)^{4}
$$

where the scaling variable $x=Q^{2} /\left(Q^{2}+M^{2}\right)$. This does not scale (i.e., is not a function of $x$ alone), but grows logarithmically with $Q^{2}$ at fixed $x$.

This behavior persists when one does the calculation to all orders in $\left.Q_{C D}{ }^{14}\right)$. The leading term behaves as $f(x)$ ln $Q^{2}$, with $f(x)$ calculable. This should be contrasted with ordinary deep inelastic scattering on a hadron, where at fixed $x$ the structure function eventually falls with increasing $Q^{2}$ due to $Q C D$ corrections and the functional form in $x$ of the structure functions is not calculable at some arbitrary initial value of $Q^{2}$. Most remarkable, the first very preliminary data on this process from the PLUTO collaboration at PETRA has just appeared and it shows the right magnitude and roughly the right shape ${ }^{12}$ ).

\section{Conclusion}

The above examples show photon-photon interactions to be a microcosm of the kind of physics questions being addressed in the world of strong interactions today. With the new generation of $\mathrm{e}^{+} \mathrm{e}^{-}$machines now running, we can look forward to some rather decisive answers in the immediate future.

\section{Acknowledgment}

This work was supported by the Department of Energy under contract number DE-AC03-76SF00515. 


\section{References}

1. L. Landau and E. Lifshitz, Physik Z. Sowjetunion 6 (1934) 244;

E. J. Williams, Kg1. Danske Videnskab. Mat. Fys. Medd. 13 (No. 4) (1934).

2. 0. Halpern, Phys. Rev. 44 (1934) 855; E. Euler, Ann. der Phys. 26 (1936) 398.

3. H. Terazawa, Rev. Mod. Phys. 45 (1973) 615, reviews the subject and literature as of the early 1970 's.

4. S. J. Brodsky et al., Phys. Rev. Lett. 25 (1970) 972;

N. Arteaga-Romero et al., C. R. Acad. Sci., B269 (1969) 153).

5. Ch. Berger et al., DESY report DESY 80/34 (1 $\overline{980)}$ unpublished.

6. F. Low, Phys. Rev. 120 (1960) 582.

7. G. S. Abrams et al., Phys. Rev. Lett. 43 (1979) 477.

8. R. Hollebeek, SL $\overline{A C}$ report SLAC-PUB-251 $\overline{7}$ (1980), unpublished talk at XVth Rencontre de Moriond.

9. E. D. Bloom, talk at the Topical Conference of the SLAC Sumner Institute on Particle Physics (1980) unpublished.

10. F. J. Gilman, Proc. 1979 Int. Conf. on Two-Photon Interactions, Lake Tahoe, 1979, ed. J. F. Gunion (University of California, Davis, 1980) p. 215, reviews production of resonances in photon-photon collisions.

11. S. J. Brodsky, Proc. 1979 Int. Conf. on Two-Photon Interactions, Lake Tahoe, 1979, ed. J. F. Gunion (University of California, Davis, 1980) p. 78, reviews QCD and jet production in two-photon interactions.

12. G. Wolf, talk at the Topical Conference of the SLAC Summer Institute on Particle Physics (1980) unpublished, reviews PETRA experimental results.

13. S. J. Brodsky and G. P. LePage, to be published and private communication.

14. W. R. Frazer, Proc. 1979 Int. Conf. on Two-Photon Interactions, Lake Tahoe, 1979, ed. J. F. Gunion (University of California, Davis, 1980) p. 105, reviews the calculations of the structure function. 